Homology, Homotopy and Applications, vol.13(2), 2011, pp.113-128

\title{
ON GROEBNER BASES AND IMMERSIONS OF GRASSMANN MANIFOLDS $G_{2, n}$
}

\author{
ZORAN Z. PETROVIĆ AND BRANISLAV I. PRVULOVIĆ
}

(communicated by Donald M. Davis)

\begin{abstract}
Mod 2 cohomology of the Grassmann manifold $G_{2, n}$ is a polynomial algebra modulo a certain well-known ideal. A Groebner basis for this ideal is obtained. Using this basis, some new immersion results for Grassmannians $G_{2, n}$ are established.
\end{abstract}

\section{Introduction}

Mod 2 cohomology of Grassmann manifolds $G_{k, n}=O(n+k) / O(n) \times O(k)$ has a rather simple description. It is the polynomial algebra on the Stiefel-Whitney classes $w_{1}, w_{2}, \ldots, w_{k}$ of the canonical vector bundle $\gamma_{k}$ over $G_{k, n}$ modulo the ideal $I_{k, n}$ generated by the dual classes $\bar{w}_{n+1}, \bar{w}_{n+2}, \ldots, \bar{w}_{n+k}$. Alas, from this description it is not at all easy to establish whether a certain cohomology class is zero or not. In [6], Monks found Groebner bases for the ideal $I_{2, n}$ in the cases $n=2^{s}-3$ and $n=2^{s}-4$. Using these bases, some new results concerning the mod 2 cohomology of $G_{2,2^{s}-3}$ and $G_{2,2^{s}-4}$ were established in that paper. Also, the author used the method of modified Postnikov towers and gave an immersion result for the spaces $G_{2,2^{s}-3}$ into $\mathbb{R}^{d}$. In [9], Shimkus improved this immersion result by the same method.

Motivated by these results, we have found a reduced Groebner basis for the ideal $I_{2, n}$ for all $n$. This result is stated in Theorem 2.7. In Corollary 2.8 we present a convenient vector space bases for $H^{*}\left(G_{2, n} ; \mathbb{Z}_{2}\right)$.

Using these bases and modified Postnikov towers, in Theorem 3.11 we generalize the immersion result established in [9] and prove that $G_{2, n}$ immerses into $\mathbb{R}^{4 n-5}$ where $n$ is any odd integer $\geqslant 7$. Our result improves upon the previously known best result (obtained by Cohen in [2]) whenever $\alpha(n)=\alpha(2 n)<5$ (where $\alpha(n)$ denotes the number of ones in the binary expansion of $n$ ).

The lower bounds for the immersion dimension of $G_{2, n}$ (which is defined by $\operatorname{imm}\left(G_{2, n}\right):=\min \left\{d \mid G_{2, n}\right.$ immerses into $\left.\mathbb{R}^{d}\right\}$ ) were established by Oproiu ([8]) using the method of the Stiefel-Whitney classes. For example, he has shown that $\operatorname{imm}\left(G_{2,2^{s-1}+1}\right) \geqslant 2^{s+1}-2$. Our result states that $\operatorname{imm}\left(G_{2,2^{s-1}+1}\right) \leqslant 2^{s+1}-1$ for $s \geqslant 4$, so it only remains to check whether $G_{2,2^{s-1}+1}$ can be immersed into $\mathbb{R}^{2^{s+1}-2}$. One more example where the lower bound from $[8]$ almost reaches the upper bound obtained in Theorem 3.11 is $G_{2,7}, 22 \leqslant \operatorname{imm}\left(G_{2,7}\right) \leqslant 23$.

Received Jan 11, 2011, revised June 27, 2011; published on October 7, 2011.

2000 Mathematics Subject Classification: 57R42, 13P10, 55S45.

Key words and phrases: Grassmanian, Groebner basis, immersion.

Article available at http://intlpress.com/HHA/v13/n2/a8 and doi:10.4310/HHA.2011.v13.n2.a8

Copyright (C) 2011, International Press. Permission to copy for private use granted. 
In addition to these main results, in Theorem 3.1 we use Groebner bases to give a simple proof of the previous result of Oproiu concerning lower bounds for $\operatorname{imm}\left(G_{2, n}\right)$.

\section{Acknowledgements}

The authors would like to thank the referee for pointing out that they had overlooked the fact that the proof of Theorem 3.11 does not go through for $n=5$.

The first author was partially supported by the Ministry of Science and Environmental Protection of Republic of Serbia Project \#174032; the second author was partially supported by the Ministry of Science and Environmental Protection of Republic of Serbia Project \#174034.

\section{Groebner bases}

For positive integer $b$ and arbitrary integer $a$, the binomial coefficient is defined by $\left(\begin{array}{l}a \\ b\end{array}\right):=\frac{a(a-1) \cdots(a-b+1)}{b !}$. Also, $\left(\begin{array}{l}a \\ 0\end{array}\right):=1$. If $b$ is a negative integer, we define $\left(\begin{array}{l}a \\ b\end{array}\right)$ to be equal to zero. Then it is easy to see that the well-known formula

$$
\left(\begin{array}{l}
a \\
b
\end{array}\right)=\left(\begin{array}{c}
a-1 \\
b
\end{array}\right)+\left(\begin{array}{l}
a-1 \\
b-1
\end{array}\right)
$$

is valid for all $a, b \in \mathbb{Z}$.

From formula (1) we deduce directly that $\left(\begin{array}{l}a \\ b\end{array}\right)+\left(\begin{array}{c}a-1 \\ b-1\end{array}\right) \equiv\left(\begin{array}{c}a-1 \\ b\end{array}\right)(\bmod 2), a, b \in \mathbb{Z}$, or equivalently $\left(\begin{array}{l}a-1 \\ b-1\end{array}\right) \equiv\left(\begin{array}{l}a \\ b\end{array}\right)+\left(\begin{array}{c}a-1 \\ b\end{array}\right)(\bmod 2), a, b \in \mathbb{Z}$.

Henceforth, all binomial coefficients are considered mod 2.

Let $G_{k, n}$ be the Grassmann manifold of unoriented $k$-dimensional vector subspaces in $\mathbb{R}^{n+k}$. It is known that the cohomology algebra $H^{*}\left(G_{k, n} ; \mathbb{Z}_{2}\right)$ is isomorphic to the quotient $\mathbb{Z}_{2}\left[w_{1}, w_{2}, \ldots, w_{k}\right] / I_{k, n}$ of the polynomial algebra $\mathbb{Z}_{2}\left[w_{1}, w_{2}, \ldots, w_{k}\right]$ by the ideal $I_{k, n}$ generated by polynomials $\bar{w}_{n+1}, \bar{w}_{n+2}, \ldots, \bar{w}_{n+k}$, which are obtained from the equation

$$
\left(1+w_{1}+w_{2}+\cdots+w_{k}\right)\left(1+\bar{w}_{1}+\bar{w}_{2}+\cdots\right)=1 .
$$

For $k=2$ (which is the case from now on), we have

$$
\begin{aligned}
1+\bar{w}_{1}+\bar{w}_{2}+\cdots & =\frac{1}{1+w_{1}+w_{2}} \\
& =\sum_{t \geqslant 0}\left(w_{1}+w_{2}\right)^{t}=\sum_{t \geqslant 0} \sum_{a+b=t}\left(\begin{array}{c}
a+b \\
a
\end{array}\right) w_{1}^{a} w_{2}^{b} \\
& =\sum_{a, b \geqslant 0}\left(\begin{array}{c}
a+b \\
a
\end{array}\right) w_{1}^{a} w_{2}^{b}
\end{aligned}
$$

By identifying the homogenous parts of (cohomological) degree $r \geqslant 0$, we obtain

$$
\bar{w}_{r}=\sum_{a+2 b=r}\left(\begin{array}{c}
a+b \\
a
\end{array}\right) w_{1}^{a} w_{2}^{b} .
$$

It is understood that $a$ and $b$ are nonnegative integers. 
We use the grlex ordering on the monomials in $\mathbb{Z}_{2}\left[w_{1}, w_{2}\right]$ with $w_{1}>w_{2}$. That is, $w_{1}^{a} w_{2}^{b} \preceq w_{1}^{c} w_{2}^{d}$ if either $a+b<c+d$ or else $a+b=c+d$ and $a \leqslant c$. Of course, we will write $w_{1}^{a} w_{2}^{b} \prec w_{1}^{c} w_{2}^{d}$ when $w_{1}^{a} w_{2}^{b} \preceq w_{1}^{c} w_{2}^{d}$ and $w_{1}^{a} w_{2}^{b} \neq w_{1}^{c} w_{2}^{d}$.

We shall prove that, with respect to this ordering, the reduced Groebner basis for the ideal $I_{2, n}=\left(\bar{w}_{n+1}, \bar{w}_{n+2}\right)$ is of the form $G=\left\{g_{0}, g_{1}, \ldots, g_{n+1}\right\}$ where $\operatorname{LT}\left(g_{m}\right)=$ $w_{1}^{n+1-m} w_{2}^{m}, 0 \leqslant m \leqslant n+1$. From this it follows immediately that a vector space basis for $H^{*}\left(G_{2, n} ; \mathbb{Z}_{2}\right)$ is the set of all monomials $w_{1}^{a} w_{2}^{b}$ such that $a+b \leqslant n$.

Let us now define the polynomials $g_{m}(0 \leqslant m \leqslant n+1)$.

Definition 2.1. For $0 \leqslant m \leqslant n+1$, let

$$
g_{m}:=\sum_{a+2 b=n+1+m}\left(\begin{array}{c}
a+b-m \\
a
\end{array}\right) w_{1}^{a} w_{2}^{b} .
$$

As before, it is understood that $a, b \geqslant 0$. Note that the (cohomological) degree of the polynomial $g_{m}$ is $n+1+m$.

By comparing with the above formula for $\bar{w}_{r}$, it is obvious that $g_{0}=\bar{w}_{n+1}$. Also,

$$
w_{2} \bar{w}_{n}=\sum_{a+2 b=n}\left(\begin{array}{c}
a+b \\
a
\end{array}\right) w_{1}^{a} w_{2}^{b+1}=\sum_{a+2 b=n+2}\left(\begin{array}{c}
a+b-1 \\
a
\end{array}\right) w_{1}^{a} w_{2}^{b}=g_{1} .
$$

The change of variable $b \mapsto b-1$ does not affect the requirement that $b \geqslant 0$ since for $b=0$ the binomial coefficient $\left(\begin{array}{c}a+b-1 \\ a\end{array}\right)=\left(\begin{array}{c}n+1 \\ n+2\end{array}\right)$ is equal to 0.

From the defining formula, one can see that $b$ must be such that $m \leqslant b \leqslant \frac{n+1+m}{2}$. Namely, $a+b-m$ cannot be negative since $a+b-m<0$ implies $a+2 b \leqslant 2(a+$ b) $<2 m \leqslant n+1+m$, contradicting the requirement that $a+2 b=n+1+m$. Now, $a+b-m$ must be $\geqslant a$ in order for $\left(\begin{array}{c}a+b-m \\ a\end{array}\right)$ to be nonzero, and we conclude that $b \geqslant$ $m$. The second inequality comes from the condition $a+2 b=n+1+m$. Therefore, we have

$$
g_{m}=\sum_{b=m}^{\left[\frac{n+1+m}{2}\right]}\left(\begin{array}{c}
n+1-b \\
b-m
\end{array}\right) w_{1}^{n+1+m-2 b} w_{2}^{b} .
$$

It is obvious that the summand obtained for $b=m$ provides the leading term $\operatorname{LT}\left(g_{m}\right)=w_{1}^{n+1-m} w_{2}^{m}$.

In order to show that $G=\left\{g_{0}, g_{1}, \ldots, g_{n+1}\right\}$ is a Groebner basis for $I_{2, n}$, we define the ideal $I_{G}:=(G)=\left(g_{0}, g_{1}, \ldots, g_{n+1}\right)$ in $\mathbb{Z}_{2}\left[w_{1}, w_{2}\right]$. As we have already noticed, $\bar{w}_{n+1}=g_{0} \in I_{G}, \bar{w}_{n+2}=w_{1} \bar{w}_{n+1}+w_{2} \bar{w}_{n}=w_{1} g_{0}+g_{1} \in I_{G}$, so $I_{2, n} \subseteq I_{G}$.

It remains to prove that $I_{G} \subseteq I_{2, n}$ and that $G$ is a Groebner basis. It turns out that the following proposition plays the crucial role in proving these facts.

Proposition 2.2. For each $m \in\{0,1, \ldots, n-1\}, w_{2} g_{m}+w_{1} g_{m+1}=g_{m+2}$. Also, we have that $w_{2} g_{n}+w_{1} g_{n+1}=0$. 
Proof. We calculate

$$
\begin{aligned}
w_{2} g_{m} & +w_{1} g_{m+1} \\
& =\sum_{a+2 b=n+1+m}\left(\begin{array}{c}
a+b-m \\
a
\end{array}\right) w_{1}^{a} w_{2}^{b+1}+\sum_{a+2 b=n+m+2}\left(\begin{array}{c}
a+b-m-1 \\
a
\end{array}\right) w_{1}^{a+1} w_{2}^{b} \\
& =\sum_{a+2 b=n+m+3}\left(\begin{array}{c}
a+b-m-1 \\
a
\end{array}\right) w_{1}^{a} w_{2}^{b}+\sum_{a+2 b=n+m+3}\left(\begin{array}{c}
a+b-m-2 \\
a-1
\end{array}\right) w_{1}^{a} w_{2}^{b} \\
& =\sum_{a+2 b=n+m+3}\left(\begin{array}{c}
a+b-m-2 \\
a
\end{array}\right) w_{1}^{a} w_{2}^{b}=g_{m+2} .
\end{aligned}
$$

We note that, for the similar reasons as above, the change of variable $b \mapsto b-1$ $(a \mapsto a-1)$ does not affect the requirement that $b \geqslant 0(a \geqslant 0)$.

The second statement is a consequence of the equalities $g_{n}=\operatorname{LT}\left(g_{n}\right)=w_{1} w_{2}^{n}$ and $g_{n+1}=\operatorname{LT}\left(g_{n+1}\right)=w_{2}^{n+1}$ which are easily seen from (2).

Corollary 2.3. $I_{G} \subseteq I_{2, n}$.

Proof. We already know that

$$
g_{0}=\bar{w}_{n+1} \in I_{2, n} \quad \text { and } \quad g_{1}=w_{1} \bar{w}_{n+1}+\bar{w}_{n+2} \in I_{2, n} .
$$

Proposition 2.2 applies, and by induction on $m$ we have that $g_{m} \in I_{2, n}(0 \leqslant m \leqslant$ $n+1)$. The corollary follows.

Therefore, $G$ is a basis for $I_{2, n}$, and we wish to prove that it is a Groebner basis. We recall that (for a fixed monomial ordering) the $S$-polynomial of polynomials $f, g \in$ $\mathbb{Z}_{2}\left[x_{1}, x_{2}, \ldots, x_{k}\right]$ is given by (we work with mod 2 coefficients)

$$
S(f, g)=\frac{L}{\mathrm{LT}(f)} \cdot f+\frac{L}{\mathrm{LT}(g)} \cdot g,
$$

where $L=\operatorname{lcm}(\operatorname{LT}(f), \operatorname{LT}(g))$ denotes the least common multiple of $\operatorname{LT}(f)$ and $\operatorname{LT}(g)$. If $0 \leqslant m<m+s \leqslant n+1$, we see that

$$
\operatorname{lcm}\left(\operatorname{LT}\left(g_{m}\right), \operatorname{LT}\left(g_{m+s}\right)\right)=\operatorname{lcm}\left(w_{1}^{n+1-m} w_{2}^{m}, w_{1}^{n+1-m-s} w_{2}^{m+s}\right)=w_{1}^{n+1-m} w_{2}^{m+s},
$$

and so we have

$$
S\left(g_{m}, g_{m+s}\right)=w_{2}^{s} g_{m}+w_{1}^{s} g_{m+s} .
$$

We are going to prove that $G$ satisfies a sufficient condition (see [1] ) for being a Groebner basis. In order to do that, we recall the following definition and theorem ([1, p. 219]). We formulate them for the field $R=\mathbb{Z}_{2}$. It is assumed that we have an ordering $\preceq$ on the monomials in $\mathbb{Z}_{2}\left[x_{1}, x_{2}, \ldots, x_{k}\right]$.

Definition 2.4. Let $F$ be a finite subset of $\mathbb{Z}_{2}\left[x_{1}, x_{2}, \ldots, x_{k}\right], f \in \mathbb{Z}_{2}\left[x_{1}, x_{2}, \ldots, x_{k}\right]$ a nonzero polynomial and $t$ a fixed monomial. If $f$ can be written as a finite sum of the form $\sum_{i} m_{i} f_{i}$, where $f_{i} \in F$ and $m_{i} \in \mathbb{Z}_{2}\left[x_{1}, x_{2}, \ldots, x_{k}\right]$ are nonzero monomials such that $\operatorname{LT}\left(m_{i} f_{i}\right) \preceq t$ for all $i$, we say that $\sum_{i} m_{i} f_{i}$ is a $t$-representation of $f$ with respect to $F$. 
Theorem 2.5. Let $F$ be a finite subset of $\mathbb{Z}_{2}\left[x_{1}, x_{2}, \ldots, x_{k}\right], 0 \notin F$. If for all $f_{1}, f_{2}$ $\in F, S\left(f_{1}, f_{2}\right)$ either equals zero or has a $t$-representation with respect to $F$ for some monomial $t \prec \operatorname{lcm}\left(\operatorname{LT}\left(f_{1}\right), \operatorname{LT}\left(f_{2}\right)\right)$, then $F$ is a Groebner basis.

We need the following lemma.

Lemma 2.6. For $0 \leqslant m<m+s \leqslant n+1, S\left(g_{m}, g_{m+s}\right)=\sum_{i=0}^{s-1} w_{1}^{i} w_{2}^{s-1-i} g_{m+2+i}$.

It is understood that for $m+s=n+1$, the last summand in this sum (for $i=$ $s-1)$ is zero.

Proof. We proceed by induction on $s$. For $s=1$, we obtain

$$
S\left(g_{m}, g_{m+1}\right)=w_{2} g_{m}+w_{1} g_{m+1}=g_{m+2}=\sum_{i=0}^{0} w_{1}^{i} w_{2}^{-i} g_{m+2+i},
$$

using (3) and Proposition 2.2. For the inductive step, we have

$$
\begin{aligned}
S\left(g_{m}, g_{m+s}\right) & =w_{2}^{s} g_{m}+w_{1}^{s} g_{m+s} \\
& =w_{2}^{s} g_{m}+w_{2} w_{1}^{s-1} g_{m+s-1}+w_{2} w_{1}^{s-1} g_{m+s-1}+w_{1}^{s} g_{m+s} \\
& =w_{2} S\left(g_{m}, g_{m+s-1}\right)+w_{1}^{s-1} g_{m+s+1} \\
& =w_{1}^{s-1} g_{m+s+1}+\sum_{i=0}^{s-2} w_{1}^{i} w_{2}^{s-1-i} g_{m+2+i} \\
& =\sum_{i=0}^{s-1} w_{1}^{i} w_{2}^{s-1-i} g_{m+2+i},
\end{aligned}
$$

again by (3), Proposition 2.2 and the induction hypothesis. It is clear that if $m+s=$ $n+1$ then the summand $w_{1}^{s-1} g_{m+s+1}$ does not appear in the sum (Proposition 2.2) and so $0 \leqslant i \leqslant s-2$ in this case.

Theorem 2.7. Let $n \geqslant 2$. Then $G=\left\{g_{0}, g_{1}, \ldots, g_{n+1}\right\}$ defined above is the reduced Groebner basis for the ideal $I_{2, n}$ in $\mathbb{Z}_{2}\left[w_{1}, w_{2}\right]$ with respect to the grlex ordering $\preceq$.

Proof. We have already shown that $G$ is a basis for $I_{2, n}$. We wish to apply Theorem 2.5. Let $g_{m}$ and $g_{m+s}(0 \leqslant m<m+s \leqslant n+1)$ be two arbitrary elements of $G$. If $m=n$, then $m+s$ must be $n+1$ and, using (3) and Proposition 2.2, one obtains $S\left(g_{m}, g_{m+s}\right)=S\left(g_{n}, g_{n+1}\right)=w_{2} g_{n}+w_{1} g_{n+1}=0$. If $m \leqslant n-1$, then according to Lemma 2.6,

$$
S\left(g_{m}, g_{m+s}\right)=\sum_{i=0}^{s-1} w_{1}^{i} w_{2}^{s-1-i} g_{m+2+i} .
$$

Define $t=t(m, s):=w_{1}^{n-1-m} w_{2}^{m+s+1}$. First of all, observe that

$$
t \prec w_{1}^{n+1-m} w_{2}^{m+s}=\operatorname{lcm}\left(\operatorname{LT}\left(g_{m}\right), \operatorname{LT}\left(g_{m+s}\right)\right) .
$$


Now, for all $i \in\{0,1, \ldots, s-1\}$,

$$
\begin{aligned}
\mathrm{LT}\left(w_{1}^{i} w_{2}^{s-1-i} g_{m+2+i}\right) & =w_{1}^{i} w_{2}^{s-1-i} \operatorname{LT}\left(g_{m+2+i}\right) \\
& =w_{1}^{i} w_{2}^{s-1-i} w_{1}^{n+1-m-2-i} w_{2}^{m+2+i} \\
& =w_{1}^{n-1-m} w_{2}^{m+s+1}=t .
\end{aligned}
$$

Theorem 2.5 applies, and we conclude that $G$ is a Groebner basis for $I_{2, n}$.

To see that it is the reduced one, we observe that $\{\mathrm{LT}(g) \mid g \in G\}$ is the set of all monomials $w_{1}^{a} w_{2}^{b}$ such that $a+b=n+1$. Also, by looking at formula (2), we see that all other terms appearing in $g_{m}$ have the sum of the exponents $<n+1$, and so they cannot be divisible by any of the leading terms in $G$.

Since $G$ is a Groebner basis for $I_{2, n}$, a vector space basis for $\mathbb{Z}_{2}\left[w_{1}, w_{2}\right] / I_{2, n}$ could be formed by taking all the monomials in $\mathbb{Z}_{2}\left[w_{1}, w_{2}\right]$ (more precisely, their classes) which are not divisible by any of $\operatorname{LT}\left(g_{0}\right), \operatorname{LT}\left(g_{1}\right), \ldots, \operatorname{LT}\left(g_{n+1}\right)$. As we have noticed in the proof of Theorem 2.7, the set $\{\operatorname{LT}(g) \mid g \in G\}$ is the set of all monomials $w_{1}^{a} w_{2}^{b}$ such that $a+b=n+1$. From this it is obvious that $w_{1}^{a} w_{2}^{b}$ is not divisible by any of the leading terms $\operatorname{LT}\left(g_{m}\right)$ if and only if $a+b \leqslant n$. By collecting all these facts, we have proved the following corollary.

Corollary 2.8. Let $n \geqslant 2$. If $w_{i}$ is the $i$-th Stiefel-Whitney class of the canonical vector bundle $\gamma_{2}$ over $G_{2, n}$, then the set $\left\{w_{1}^{a} w_{2}^{b} \mid a+b \leqslant n\right\}$ is a vector space basis for $H^{*}\left(G_{2, n} ; \mathbb{Z}_{2}\right)$.

Let us now determine a few elements of the Groebner basis $G$ which will be used in our later calculations. As we have already noticed, by formula (2), $g_{n+1}=w_{2}^{n+1}$ and $g_{n}=w_{1} w_{2}^{n}$. Using this and Proposition 2.2, we obtain $w_{2} g_{n-1}=w_{1} g_{n}+g_{n+1}=$ $w_{1}^{2} w_{2}^{n}+w_{2}^{n+1}=w_{2}\left(w_{1}^{2} w_{2}^{n-1}+w_{2}^{n}\right)$, and so we deduce that $g_{n-1}=w_{1}^{2} w_{2}^{n-1}+w_{2}^{n}$. Continuing in the same manner, one gets

$$
\begin{aligned}
& g_{n-2}=w_{1}^{3} w_{2}^{n-2} ; \\
& g_{n-3}=w_{1}^{4} w_{2}^{n-3}+w_{1}^{2} w_{2}^{n-2}+w_{2}^{n-1} ; \\
& g_{n-4}=w_{1}^{5} w_{2}^{n-4}+w_{1} w_{2}^{n-2} ; \\
& g_{n-5}=w_{1}^{6} w_{2}^{n-5}+w_{1}^{4} w_{2}^{n-4}+w_{2}^{n-2} .
\end{aligned}
$$

\section{Immersions}

In order to construct the immersions of Grassmannians $G_{2, n}$ into Euclidean spaces, we recall the theorem of Hirsch $([4])$ which states that a smooth compact $m$-manifold $M^{m}$ immerses in $\mathbb{R}^{m+l}$ if and only if the classifying map $f_{\nu}: M^{m} \rightarrow B O$ of the stable normal bundle $\nu$ of $M^{m}$ lifts up to $B O(l)$.

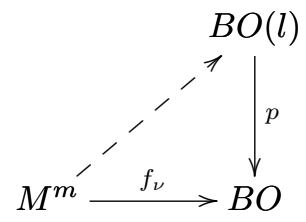


Let $\operatorname{imm}\left(M^{m}\right)$ denote the least integer $d$ such that $M^{m}$ immerses into $\mathbb{R}^{d}$. By Hirsch's theorem, if $w_{k}(\nu) \neq 0$ then $\operatorname{imm}\left(M^{m}\right) \geqslant m+k$.

As in Corollary 2.8, let $w_{i}$ be the $i$-th Stiefel-Whitney class of the canonical vector bundle $\gamma_{2}$ over $G_{2, n}(n \geqslant 2)$. It is well known (see [8, p. 179]) that, if $2^{s}$ is the least power of 2 exceeding $n$, i.e., $2^{s-1} \leqslant n<2^{s}$, then for the total Stiefel-Whitney class $w(\nu)$ of the stable normal bundle $\nu$ of $G_{2, n}$, one has

$$
w(\nu)=\left(1+w_{1}^{2}\right)\left(1+w_{1}+w_{2}\right)^{2^{s+1}-2-n} .
$$

For $n=2^{s}-2$, from formula (2) we have that

$$
g_{0}=\sum_{b=0}^{2^{s-1}-1}\left(\begin{array}{c}
2^{s}-1-b \\
b
\end{array}\right) w_{1}^{2^{s}-1-2 b} w_{2}^{b}=w_{1}^{2^{s}-1}
$$

since the binomial coefficient $\left(\begin{array}{c}2^{s}-1-b \\ b\end{array}\right)$ is odd only for $b=0$ (by Lucas formula). This means that $w_{1}^{2^{s}-1}=0$ in $H^{*}\left(G_{2,2^{s}-2} ; \mathbb{Z}_{2}\right)$. But then $w_{1}^{2^{s}-1}=0$ in $H^{*}\left(G_{2, n} ; \mathbb{Z}_{2}\right)$ for all $n \leqslant 2^{s}-2$ since the inclusion $i: G_{2, n} \rightarrow G_{2,2^{s}-2}$ is obviously covered by a map of canonical bundles $\gamma_{2}$.

If $2^{s-1} \leqslant n \leqslant 2^{s}-2$, then by formula (4) we have

$$
\begin{aligned}
w(\nu) & =\left(1+w_{1}^{2}\right)\left(1+w_{1}+w_{2}\right)^{2^{s}}\left(1+w_{1}+w_{2}\right)^{2^{s}-2-n} \\
& =\left(1+w_{1}^{2}\right)\left(1+w_{1}^{2^{s}}+w_{2}^{2^{s}}\right)\left(1+w_{1}+w_{2}\right)^{2^{s}-2-n} .
\end{aligned}
$$

Now, $w_{2}^{2^{s}}=0$ because it is a class of degree $2^{s+1}>2^{s+1}-4 \geqslant 2 n=\operatorname{dim}\left(G_{2, n}\right)$. Also, by the previous discussion $w_{1}^{2^{s}}=0$ and (4) simplifies to

$$
w(\nu)=\left(1+w_{1}^{2}\right)\left(1+w_{1}+w_{2}\right)^{2^{s}-2-n} .
$$

If $n=2^{s}-1$, then from (4) we obtain

$$
\begin{aligned}
w(\nu) & =\left(1+w_{1}^{2}\right)\left(1+w_{1}+w_{2}\right)^{2^{s}-1} \\
& =\left(1+w_{1}\right)^{2} \sum_{i=0}^{2^{s}-1}\left(\begin{array}{c}
2^{s}-1 \\
i
\end{array}\right)\left(1+w_{1}\right)^{i} w_{2}^{2^{s}-1-i} \\
& =\sum_{i=0}^{2^{s}-1}\left(1+w_{1}\right)^{i+2} w_{2}^{2^{s}-1-i} \\
& =\sum_{i=0}^{2^{s}-1} \sum_{j=0}^{i+2}\left(\begin{array}{c}
i+2 \\
j
\end{array}\right) w_{1}^{j} w_{2}^{2^{s}-1-i} .
\end{aligned}
$$

We now recall a theorem of Oproiu ([8]), and we prove it using the Groebner basis from Theorem 2.7.

Theorem 3.1 (Oproiu [8]). For $2 \leqslant 2^{s-1} \leqslant n<2^{s}$, we have:

(a) If $n \leqslant 2^{s}-2$, then $\operatorname{imm}\left(G_{2, n}\right) \geqslant 2^{s+1}-2$.

(b) $\operatorname{imm}\left(G_{2,2^{s}-1}\right) \geqslant 3 \cdot 2^{s}-2$.

Proof. (a) The top class in the expression (5) is $w_{2^{s+1}-2-2 n}(\nu)=w_{1}^{2} w_{2}^{2^{s}-2-n}$, and since the sum of the exponents $2+2^{s}-2-n=2^{s}-n \leqslant 2^{s-1} \leqslant n$, by Corollary 2.8 
we have that $w_{2^{s+1}-2-2 n}(\nu) \neq 0$ and we conclude that

$$
\operatorname{imm}\left(G_{2, n}\right) \geqslant \operatorname{dim}\left(G_{2, n}\right)+2^{s+1}-2-2 n=2^{s+1}-2 .
$$

(b) From the equality (6) we calculate

$$
\begin{aligned}
w_{2^{s}}(\nu) & =\sum_{i=2^{s-1}-1}^{2^{s}-1}\left(\begin{array}{c}
i+2 \\
2 i+2-2^{s}
\end{array}\right) w_{1}^{2 i+2-2^{s}} w_{2}^{2^{s}-1-i} \\
& =\sum_{l=0}^{2^{s-1}}\left(\begin{array}{c}
2^{s}+1-l \\
2^{s}-2 l
\end{array}\right) w_{1}^{2^{s}-2 l} w_{2}^{l}=\sum_{l=0}^{2^{s-1}}\left(\begin{array}{c}
2^{s}+1-l \\
l+1
\end{array}\right) w_{1}^{2^{s}-2 l} w_{2}^{l} \\
& =\left(\begin{array}{c}
2^{s}+1 \\
1
\end{array}\right) w_{1}^{2^{s}}+\left(\begin{array}{c}
2^{s} \\
2
\end{array}\right) w_{1}^{2^{s}-2} w_{2}+\left(\begin{array}{c}
2^{s}-1 \\
3
\end{array}\right) w_{1}^{2^{s}-4} w_{2}^{2}+\cdots \\
& =w_{1}^{2^{s}}+w_{1}^{2^{s}-4} w_{2}^{2}+\cdots,
\end{aligned}
$$

where the unwritten monomials (if there are any) have the sum of the exponents $\leqslant 2^{s}-3=n-2$. Note that, since $2^{s-1} \geqslant 2$, three written summands must appear in the sum.

On the other hand, from the equality (2) we see that the first element of the Groebner basis in this case is

$$
\begin{aligned}
g_{0} & =\sum_{b=0}^{2^{s-1}}\left(\begin{array}{c}
2^{s}-b \\
b
\end{array}\right) w_{1}^{2^{s}-2 b} w_{2}^{b}=w_{1}^{2^{s}}+\left(2^{s}-1\right) w_{1}^{2^{s}-2} w_{2}+\left(\begin{array}{c}
2^{s}-2 \\
2
\end{array}\right) w_{1}^{2^{s}-4} w_{2}^{2}+\cdots \\
& =w_{1}^{2^{s}}+w_{1}^{2^{s}-2} w_{2}+w_{1}^{2^{s}-4} w_{2}^{2}+\cdots .
\end{aligned}
$$

Again, the unwritten monomials have the sum of the exponents $\leqslant n-2$, and three written ones must be here.

By adding these two equalities together, using the fact that $g_{0}=0$ in $H^{*}\left(G_{2, n} ; \mathbb{Z}_{2}\right)$ we obtain

$$
w_{2^{s}}(\nu)=w_{1}^{2^{s}-2} w_{2}+\cdots .
$$

The sum of the exponents in the monomial $w_{1}^{2^{s}-2} w_{2}$ is $2^{s}-1=n$, and in the remaining monomials (if there are any) this sum is $\leqslant n-2$, so none of these monomials is divisible by any of the leading terms $\operatorname{LT}\left(g_{m}\right)$. This means that we have obtained the remainder of dividing $w_{2^{s}}(\nu)$ by $G$. Since $w_{1}^{2^{s}-2} w_{2}$ must appear in this remainder, we conclude that $w_{2^{s}}(\nu) \neq 0$. Finally, this implies that

$$
\operatorname{imm}\left(G_{2,2^{s}-1}\right) \geqslant \operatorname{dim}\left(G_{2,2^{s}-1}\right)+2^{s}=3 \cdot 2^{s}-2
$$

and we are done.

Example 3.2. If $n=2^{s-1}>2$, then $\operatorname{imm}\left(G_{2,2^{s-1}}\right) \geqslant 2^{s+1}-2=2 \cdot \operatorname{dim}\left(G_{2,2^{s-1}}\right)-2$. By the result of Massey [5, Theorem V], if $M^{m}$ is orientable, $m>4$ and $w_{2}(\nu)$. $w_{m-2}(\nu)=0$, then $M^{m}$ immerses into $\mathbb{R}^{2 m-2}$. Now, $G_{2,2^{s-1}}$ is orientable (Grassmannian $G_{k, n}$ is orientable if and only if $n+k$ is even; see [8, p. 179]), and from formula (5) we have

$$
w(\nu)=\left(1+w_{1}^{2}\right)\left(1+w_{1}+w_{2}\right)^{2^{s-1}-2},
$$


so

$$
w_{2}(\nu)=\left(1+\left(\begin{array}{c}
2^{s-1}-2 \\
2
\end{array}\right)\right) w_{1}^{2}+\left(2^{s-1}-2\right) w_{2}=0
$$

since $2^{s-1}>2$. This implies that $G_{2,2^{s-1}}$ immerses into $\mathbb{R}^{2^{s+1}-2}$, i.e., $\operatorname{imm}\left(G_{2,2^{s-1}}\right) \leqslant$ $2^{s+1}-2$, so for $2^{s-1}>2$, we actually have the equality

$$
\operatorname{imm}\left(G_{2,2^{s-1}}\right)=2^{s+1}-2 .
$$

Also, we note that for $G_{2,3}$, Oproiu's Theorem $3.1(\mathrm{~b})$ gives $\operatorname{imm}\left(G_{2,3}\right) \geqslant 10$, and by Cohen's theorem $([2]), \operatorname{imm}\left(G_{2,3}\right) \leqslant 10$, so $\operatorname{imm}\left(G_{2,3}\right)=10$. For $G_{2,5}$, the results of Oproiu ([8]) and Monks $([\mathbf{6}])$ provide inequalities $14 \leqslant \operatorname{imm}\left(G_{2,5}\right) \leqslant 17$.

We now turn to the proof of the immersion result.

Lemma 3.3. Let $n$ be an odd integer $\geqslant 5$. For the stable normal bundle $\nu$ of $G_{2, n}$ we have:

(a) $w_{i}(\nu)=0$ for $i \geqslant 2 n-5$;

(b) $w_{1}(\nu)=w_{1}$;

(c) $w_{2}(\nu)=w_{2}$ if $n \equiv 3(\bmod 4) ; w_{2}(\nu)=w_{1}^{2}+w_{2}$ if $n \equiv 1(\bmod 4)$.

Proof. As above, let $s$ be the integer such that $2^{s-1} \leqslant n<2^{s}$. Since $n$ is odd, we have that $n \geqslant 2^{s-1}+1$. This implies $4 n \geqslant 2^{s+1}+4$, i.e., $2^{s+1}-2-2 n<2 n-5$.

If $n \neq 2^{s}-1$, then from formula (5) we see that the top class in the expression for $w(\nu)$, namely $w_{1}^{2} w_{2}^{2^{s}-2-n}$, is of degree $2^{s+1}-2-2 n$, and by the previous inequality, we deduce that $w_{i}(\nu)=0$ for $i \geqslant 2 n-5$.

For $n=2^{s}-1$, Oproiu shows [8, p. 182] that the top nonzero class in the expression (4) is of degree $2^{s}$ and, since $n \geqslant 7$ in this case, we conclude that $2^{s}=n+1<$ $2 n-5$ obtaining (a).

From formula (4) we read off

$$
\begin{gathered}
w_{1}(\nu)=\left(2^{s+1}-2-n\right) w_{1} \\
w_{2}(\nu)=\left(1+\left(\begin{array}{c}
2^{s+1}-2-n \\
2
\end{array}\right)\right) w_{1}^{2}+\left(2^{s+1}-2-n\right) w_{2}
\end{gathered}
$$

and obtain (b) and (c).

A few more lemmas will be useful.

Lemma 3.4. In $H^{*}\left(G_{2, n} ; \mathbb{Z}_{2}\right)$, for all nonnegative integers a and $b$, the following relations hold:

(a) $S q^{1}\left(w_{1}^{a} w_{2}^{b}\right)=(a+b) w_{1}^{a+1} w_{2}^{b}$;

(b) $S q^{2}\left(w_{1}^{a} w_{2}^{b}\right)=b w_{1}^{a} w_{2}^{b+1}+\left(\begin{array}{c}a+b \\ 2\end{array}\right) w_{1}^{a+2} w_{2}^{b}$.

Proof. Since $S q^{j}\left(w_{1}^{a}\right)=\left(\begin{array}{c}a \\ j\end{array}\right) w_{1}^{a+j}$, the formulas are true for $b=0$. We proceed by induction on $b$.

(a) By the $\mathrm{Wu}$ formula, $S q^{1} w_{2}=w_{1} w_{2}$. Using the Cartan formula and the induction hypothesis, we have 


$$
\begin{aligned}
S q^{1}\left(w_{1}^{a} w_{2}^{b}\right) & =S q^{1}\left(w_{2} w_{1}^{a} w_{2}^{b-1}\right) \\
& =w_{1} w_{2} w_{1}^{a} w_{2}^{b-1}+(a+b-1) w_{2} w_{1}^{a+1} w_{2}^{b-1} \\
& =(a+b) w_{1}^{a+1} w_{2}^{b} .
\end{aligned}
$$

(b) For the induction step we use again formulas of Cartan and $\mathrm{Wu}$, the statement (a) and the fact that $S q^{2} w_{2}=w_{2}^{2}$. We calculate

$$
\begin{aligned}
S q^{2}\left(w_{1}^{a} w_{2}^{b}\right)= & S q^{2}\left(w_{2} w_{1}^{a} w_{2}^{b-1}\right) \\
= & w_{1}^{a} w_{2}^{b+1}+(a+b-1) w_{1}^{a+2} w_{2}^{b}+(b-1) w_{1}^{a} w_{2}^{b+1} \\
& \quad+\left(\begin{array}{c}
a+b-1 \\
2
\end{array}\right) w_{1}^{a+2} w_{2}^{b} \\
= & b w_{1}^{a} w_{2}^{b+1}+\left(\begin{array}{c}
a+b \\
2
\end{array}\right) w_{1}^{a+2} w_{2}^{b},
\end{aligned}
$$

and the proof is complete.

Lemma 3.5. The map $\left(S q^{2}+w_{2}(\nu)\right): H^{2 n-5}\left(G_{2, n} ; \mathbb{Z}_{2}\right) \rightarrow H^{2 n-3}\left(G_{2, n} ; \mathbb{Z}_{2}\right)$, where $n$ is an odd integer $\geqslant 5$, is determined by the equalities

$$
\begin{aligned}
\left(S q^{2}+w_{2}(\nu)\right)\left(w_{1}^{5} w_{2}^{n-5}\right) & =\left(S q^{2}+w_{2}(\nu)\right)\left(w_{1}^{3} w_{2}^{n-4}\right)=\left(S q^{2}+w_{2}(\nu)\right)\left(w_{1} w_{2}^{n-3}\right) \\
& =w_{1} w_{2}^{n-2} .
\end{aligned}
$$

Proof. By Corollary 2.8, the set $\left\{w_{1}^{5} w_{2}^{n-5}, w_{1}^{3} w_{2}^{n-4}, w_{1} w_{2}^{n-3}\right\}$ is a vector space basis for $H^{2 n-5}\left(G_{2, n} ; \mathbb{Z}_{2}\right)$.

Now, if $n \equiv 3(\bmod 4)$, using Lemma 3.3, Lemma 3.4 and Groebner basis from Theorem 2.7, we calculate

$$
\begin{aligned}
\left(S q^{2}+w_{2}(\nu)\right)\left(w_{1}^{5} w_{2}^{n-5}\right) & =S q^{2}\left(w_{1}^{5} w_{2}^{n-5}\right)+w_{2} w_{1}^{5} w_{2}^{n-5} \\
& =(n-5) w_{1}^{5} w_{2}^{n-4}+\left(\begin{array}{c}
n \\
2
\end{array}\right) w_{1}^{7} w_{2}^{n-5}+w_{1}^{5} w_{2}^{n-4} \\
& =w_{1}^{7} w_{2}^{n-5}+w_{1}^{5} w_{2}^{n-4} \\
& =w_{1}\left(g_{n-5}+w_{1}^{4} w_{2}^{n-4}+w_{2}^{n-2}\right)+w_{1}^{5} w_{2}^{n-4}=w_{1} w_{2}^{n-2} ; \\
\left(S q^{2}+w_{2}(\nu)\right)\left(w_{1}^{3} w_{2}^{n-4}\right) & =S q^{2}\left(w_{1}^{3} w_{2}^{n-4}\right)+w_{2} w_{1}^{3} w_{2}^{n-4} \\
& =(n-4) w_{1}^{3} w_{2}^{n-3}+\left(\begin{array}{c}
n-1 \\
2
\end{array}\right) w_{1}^{5} w_{2}^{n-4}+w_{1}^{3} w_{2}^{n-3} \\
& =w_{1}^{5} w_{2}^{n-4}=g_{n-4}+w_{1} w_{2}^{n-2}=w_{1} w_{2}^{n-2} ; \\
\left(S q^{2}+w_{2}(\nu)\right)\left(w_{1} w_{2}^{n-3}\right) & =S q^{2}\left(w_{1} w_{2}^{n-3}\right)+w_{2} w_{1} w_{2}^{n-3} \\
& =(n-3) w_{1} w_{2}^{n-2}+\left(\begin{array}{c}
n-2 \\
2
\end{array}\right) w_{1}^{3} w_{2}^{n-3}+w_{1} w_{2}^{n-2} \\
& =w_{1} w_{2}^{n-2} .
\end{aligned}
$$


Similarly, if $n \equiv 1(\bmod 4)$, we have

$$
\begin{aligned}
\left(S q^{2}+w_{2}(\nu)\right)\left(w_{1}^{5} w_{2}^{n-5}\right) & =S q^{2}\left(w_{1}^{5} w_{2}^{n-5}\right)+w_{1}^{2} w_{1}^{5} w_{2}^{n-5}+w_{2} w_{1}^{5} w_{2}^{n-5} \\
& =(n-5) w_{1}^{5} w_{2}^{n-4}+\left(\begin{array}{c}
n \\
2
\end{array}\right) w_{1}^{7} w_{2}^{n-5}+w_{1}^{7} w_{2}^{n-5}+w_{1}^{5} w_{2}^{n-4} \\
& =w_{1}^{7} w_{2}^{n-5}+w_{1}^{5} w_{2}^{n-4} \\
& =w_{1}\left(g_{n-5}+w_{1}^{4} w_{2}^{n-4}+w_{2}^{n-2}\right)+w_{1}^{5} w_{2}^{n-4}=w_{1} w_{2}^{n-2} ; \\
\left(S q^{2}+w_{2}(\nu)\right)\left(w_{1}^{3} w_{2}^{n-4}\right) & =S q^{2}\left(w_{1}^{3} w_{2}^{n-4}\right)+w_{1}^{2} w_{1}^{3} w_{2}^{n-4}+w_{2} w_{1}^{3} w_{2}^{n-4} \\
& =(n-4) w_{1}^{3} w_{2}^{n-3}+\left(\begin{array}{c}
n-1 \\
2
\end{array}\right) w_{1}^{5} w_{2}^{n-4}+w_{1}^{5} w_{2}^{n-4}+w_{1}^{3} w_{2}^{n-3} \\
& =w_{1}^{5} w_{2}^{n-4}=g_{n-4}+w_{1} w_{2}^{n-2}=w_{1} w_{2}^{n-2} ; \\
\left(S q^{2}+w_{2}(\nu)\right)\left(w_{1} w_{2}^{n-3}\right) & =S q^{2}\left(w_{1} w_{2}^{n-3}\right)+w_{1}^{2} w_{1} w_{2}^{n-3}+w_{2} w_{1} w_{2}^{n-3} \\
& =(n-3) w_{1} w_{2}^{n-2}+\left(\begin{array}{c}
n-2 \\
2
\end{array}\right) w_{1}^{3} w_{2}^{n-3}+w_{1}^{3} w_{2}^{n-3}+w_{1} w_{2}^{n-2} \\
& =w_{1} w_{2}^{n-2},
\end{aligned}
$$

and the proof of the lemma is complete.

Lemma 3.6. The map $S q^{1}: H^{2 n-2}\left(G_{2, n} ; \mathbb{Z}_{2}\right) \rightarrow H^{2 n-1}\left(G_{2, n} ; \mathbb{Z}_{2}\right)$, where $n$ is an odd integer $\geqslant 5$, is trivial.

Proof. The set $\left\{w_{1}^{2} w_{2}^{n-2}, w_{2}^{n-1}\right\}$ is a vector space basis for $H^{2 n-2}\left(G_{2, n} ; \mathbb{Z}_{2}\right)$ (Corollary 2.8). Using Lemma 3.4, we obtain

$$
\begin{gathered}
S q^{1}\left(w_{1}^{2} w_{2}^{n-2}\right)=n w_{1}^{3} w_{2}^{n-2}=w_{1}^{3} w_{2}^{n-2}=g_{n-2}=0 ; \\
S q^{1}\left(w_{2}^{n-1}\right)=(n-1) w_{1} w_{2}^{n-1}=0,
\end{gathered}
$$

which proves the lemma.

Lemma 3.7. The map $\left(S q^{2}+w_{2}(\nu)\right): H^{2 n-3}\left(G_{2, n} ; \mathbb{Z}_{2}\right) \rightarrow H^{2 n-1}\left(G_{2, n} ; \mathbb{Z}_{2}\right)$, where $n$ is an odd integer $\geqslant 5$, is determined by the equalities:

$$
\begin{aligned}
& \left(S q^{2}+w_{2}(\nu)\right)\left(w_{1}^{3} w_{2}^{n-3}\right)=w_{1} w_{2}^{n-1} \neq 0 ; \\
& \left(S q^{2}+w_{2}(\nu)\right)\left(w_{1} w_{2}^{n-2}\right)=0 .
\end{aligned}
$$

Proof. Again by Corollary 2.8, the classes $w_{1}^{3} w_{2}^{n-3}$ and $w_{1} w_{2}^{n-2}$ form a vector space basis for $H^{2 n-3}\left(G_{2, n} ; \mathbb{Z}_{2}\right)$, and the class $w_{1} w_{2}^{n-1}$ is nontrivial in $H^{2 n-1}\left(G_{2, n} ; \mathbb{Z}_{2}\right) \cong$ $\mathbb{Z}_{2}$.

By Lemma 3.3 and Lemma 3.4, for $n \equiv 3(\bmod 4)$ we have

$$
\begin{aligned}
\left(S q^{2}+w_{2}(\nu)\right)\left(w_{1}^{3} w_{2}^{n-3}\right) & =S q^{2}\left(w_{1}^{3} w_{2}^{n-3}\right)+w_{2} w_{1}^{3} w_{2}^{n-3} \\
& =(n-3) w_{1}^{3} w_{2}^{n-2}+\left(\begin{array}{c}
n \\
2
\end{array}\right) w_{1}^{5} w_{2}^{n-3}+w_{1}^{3} w_{2}^{n-2} \\
& =w_{1}^{5} w_{2}^{n-3}+w_{1}^{3} w_{2}^{n-2} \\
& =w_{1}\left(g_{n-3}+w_{1}^{2} w_{2}^{n-2}+w_{2}^{n-1}\right)+w_{1}^{3} w_{2}^{n-2} \\
& =w_{1} w_{2}^{n-1}
\end{aligned}
$$




$$
\begin{aligned}
\left(S q^{2}+w_{2}(\nu)\right)\left(w_{1} w_{2}^{n-2}\right) & =S q^{2}\left(w_{1} w_{2}^{n-2}\right)+w_{2} w_{1} w_{2}^{n-2} \\
& =(n-2) w_{1} w_{2}^{n-1}+\left(\begin{array}{c}
n-1 \\
2
\end{array}\right) w_{1}^{3} w_{2}^{n-2}+w_{1} w_{2}^{n-1} \\
& =w_{1}^{3} w_{2}^{n-2}=g_{n-2}=0 .
\end{aligned}
$$

Likewise, for $n \equiv 1(\bmod 4)$, we obtain

$$
\begin{aligned}
\left(S q^{2}+w_{2}(\nu)\right)\left(w_{1}^{3} w_{2}^{n-3}\right) & =S q^{2}\left(w_{1}^{3} w_{2}^{n-3}\right)+w_{1}^{2} w_{1}^{3} w_{2}^{n-3}+w_{2} w_{1}^{3} w_{2}^{n-3} \\
& =(n-3) w_{1}^{3} w_{2}^{n-2}+\left(\begin{array}{c}
n \\
2
\end{array}\right) w_{1}^{5} w_{2}^{n-3}+w_{1}^{5} w_{2}^{n-3}+w_{1}^{3} w_{2}^{n-2} \\
& =w_{1}^{5} w_{2}^{n-3}+w_{1}^{3} w_{2}^{n-2} \\
& =w_{1}\left(g_{n-3}+w_{1}^{2} w_{2}^{n-2}+w_{2}^{n-1}\right)+w_{1}^{3} w_{2}^{n-2} \\
& =w_{1} w_{2}^{n-1} ; \\
\left(S q^{2}+w_{2}(\nu)\right)\left(w_{1} w_{2}^{n-2}\right) & =S q^{2}\left(w_{1} w_{2}^{n-2}\right)+w_{1}^{2} w_{1} w_{2}^{n-2}+w_{2} w_{1} w_{2}^{n-2} \\
& =(n-2) w_{1} w_{2}^{n-1}+\left(\begin{array}{c}
n-1 \\
2
\end{array}\right) w_{1}^{3} w_{2}^{n-2}+w_{1}^{3} w_{2}^{n-2}+w_{1} w_{2}^{n-1} \\
& =w_{1}^{3} w_{2}^{n-2}=g_{n-2}=0,
\end{aligned}
$$

which was to be proved.

Lemma 3.8. The map $S q^{1}: H^{2 n-3}\left(G_{2, n} ; \mathbb{Z}_{2}\right) \rightarrow H^{2 n-2}\left(G_{2, n} ; \mathbb{Z}_{2}\right)$, where $n$ is an odd integer $\geqslant 5$, is given by the equalities:

$$
\begin{aligned}
& S q^{1}\left(w_{1}^{3} w_{2}^{n-3}\right)=w_{1}^{2} w_{2}^{n-2}+w_{2}^{n-1} \\
& S q^{1}\left(w_{1} w_{2}^{n-2}\right)=0 .
\end{aligned}
$$

Proof. As we have noticed in the proof of the previous lemma, the set

$$
\left\{w_{1}^{3} w_{2}^{n-3}, w_{1} w_{2}^{n-2}\right\}
$$

is a vector space basis for $H^{2 n-3}\left(G_{2, n} ; \mathbb{Z}_{2}\right)$. So, we calculate

$$
\begin{aligned}
S q^{1}\left(w_{1}^{3} w_{2}^{n-3}\right)=n w_{1}^{4} w_{2}^{n-3}=w_{1}^{4} w_{2}^{n-3} & =g_{n-3}+w_{1}^{2} w_{2}^{n-2}+w_{2}^{n-1}=w_{1}^{2} w_{2}^{n-2}+w_{2}^{n-1}, \\
S q^{1}\left(w_{1} w_{2}^{n-2}\right) & =(n-1) w_{1}^{2} w_{2}^{n-2}=0
\end{aligned}
$$

by Lemma 3.4.

Lemma 3.9. If $n$ is an odd integer $\geqslant 5$, then in $H^{*}\left(G_{2, n} ; \mathbb{Z}_{2}\right)$ we have

$$
\left(S q^{2}+w_{1}(\nu)^{2}+w_{2}(\nu)\right) S q^{1}\left(w_{1}^{3} w_{2}^{n-4}+w_{1} w_{2}^{n-3}\right)=w_{1}^{2} w_{2}^{n-2} .
$$

Proof. By Lemma 3.4(a),

$$
S q^{1}\left(w_{1}^{3} w_{2}^{n-4}+w_{1} w_{2}^{n-3}\right)=(n-1) w_{1}^{4} w_{2}^{n-4}+(n-2) w_{1}^{2} w_{2}^{n-3}=w_{1}^{2} w_{2}^{n-3} .
$$

If $n \equiv 3(\bmod 4)$, by Lemma 3.3 and Lemma $3.4(\mathrm{~b})$, one obtains

$$
\begin{gathered}
\left(S q^{2}+w_{1}(\nu)^{2}+w_{2}(\nu)\right)\left(w_{1}^{2} w_{2}^{n-3}\right)=S q^{2}\left(w_{1}^{2} w_{2}^{n-3}\right)+w_{1}^{2} w_{1}^{2} w_{2}^{n-3}+w_{2} w_{1}^{2} w_{2}^{n-3} \\
=(n-3) w_{1}^{2} w_{2}^{n-2}+\left(\begin{array}{c}
n-1 \\
2
\end{array}\right) w_{1}^{4} w_{2}^{n-3}+w_{1}^{4} w_{2}^{n-3} \\
+w_{1}^{2} w_{2}^{n-2}=w_{1}^{2} w_{2}^{n-2} .
\end{gathered}
$$


If $n \equiv 1(\bmod 4)$, again by Lemma 3.3 and Lemma 3.4(b), we have

$$
\begin{aligned}
\left(S q^{2}+w_{1}(\nu)^{2}+w_{2}(\nu)\right)\left(w_{1}^{2} w_{2}^{n-3}\right) & =S q^{2}\left(w_{1}^{2} w_{2}^{n-3}\right)+w_{2} w_{1}^{2} w_{2}^{n-3} \\
& =(n-3) w_{1}^{2} w_{2}^{n-2}+\left(\begin{array}{c}
n-1 \\
2
\end{array}\right) w_{1}^{4} w_{2}^{n-3}+w_{1}^{2} w_{2}^{n-2} \\
& =w_{1}^{2} w_{2}^{n-2},
\end{aligned}
$$

and we are done.

Lemma 3.10. If $n$ is an odd integer $\geqslant 5$, then in $H^{*}\left(G_{2, n} ; \mathbb{Z}_{2}\right)$ the following equality holds:

$$
\left(S q^{2}+w_{2}(\nu)\right)\left(w_{1}^{2} w_{2}^{n-3}\right)=w_{2}^{n-1} .
$$

Proof. As before, we use Lemma 3.3, Lemma 3.4 and Groebner basis from Theorem 2.7.

If $n \equiv 3(\bmod 4)$,

$$
\begin{aligned}
\left(S q^{2}+w_{2}(\nu)\right)\left(w_{1}^{2} w_{2}^{n-3}\right) & =S q^{2}\left(w_{1}^{2} w_{2}^{n-3}\right)+w_{2} w_{1}^{2} w_{2}^{n-3} \\
& =(n-3) w_{1}^{2} w_{2}^{n-2}+\left(\begin{array}{c}
n-1 \\
2
\end{array}\right) w_{1}^{4} w_{2}^{n-3}+w_{1}^{2} w_{2}^{n-2} \\
& =w_{1}^{4} w_{2}^{n-3}+w_{1}^{2} w_{2}^{n-2}=g_{n-3}+w_{2}^{n-1}=w_{2}^{n-1} .
\end{aligned}
$$

If $n \equiv 1(\bmod 4)$,

$$
\begin{aligned}
\left(S q^{2}+w_{2}(\nu)\right)\left(w_{1}^{2} w_{2}^{n-3}\right) & =S q^{2}\left(w_{1}^{2} w_{2}^{n-3}\right)+w_{1}^{2} w_{1}^{2} w_{2}^{n-3}+w_{2} w_{1}^{2} w_{2}^{n-3} \\
& =(n-3) w_{1}^{2} w_{2}^{n-2}+\left(\begin{array}{c}
n-1 \\
2
\end{array}\right) w_{1}^{4} w_{2}^{n-3}+w_{1}^{4} w_{2}^{n-3}+w_{1}^{2} w_{2}^{n-2} \\
& =w_{1}^{4} w_{2}^{n-3}+w_{1}^{2} w_{2}^{n-2}=g_{n-3}+w_{2}^{n-1}=w_{2}^{n-1},
\end{aligned}
$$

completing the proof.

We are now ready to prove our immersion result.

Theorem 3.11. If $n$ is an odd integer $\geqslant 7$, then $G_{2, n}$ immerses into $\mathbb{R}^{4 n-5}$.

Proof. Let $f_{\nu}: G_{2, n} \rightarrow B O$ be the classifying map for the stable normal bundle $\nu$ of $G_{2, n}$. We want to show that $f_{\nu}$ can be lifted up to $B O(2 n-5)$. We will use the $2 n$ MPT for the fibration $p: B O(2 n-5) \rightarrow B O$ which can be constructed by the method of Gitler and Mahowald ([3]) using the result of Nussbaum ([7]) who proved that their method is applicable to the fibrations $p: B O(l) \rightarrow B O$ when $l$ is odd. The tower is presented in Figure 1 ( $K_{m}$ stands for the Eilenberg-MacLane space $K\left(\mathbb{Z}_{2}, m\right)$ ).

The relations that produce the $k$-invariants are

$$
\begin{aligned}
& k_{1}^{2}:\left(S q^{2}+w_{2}\right) w_{2 n-4}=0, \\
& k_{2}^{2}:\left(S q^{2}+w_{1}^{2}+w_{2}\right) S q^{1} w_{2 n-4}+S q^{1} w_{2 n-2}=0, \\
& k_{3}^{2}: \begin{cases}\left(S q^{4}+w_{4}\right) w_{2 n-4}+w_{2} w_{2 n-2}=0, & n \equiv 3(\bmod 4) \\
\left(S q^{4}+w_{4}\right) w_{2 n-4}+S q^{2} w_{2 n-2}=0, & n \equiv 1(\bmod 4),\end{cases} \\
& k_{1}^{3}:\left(S q^{2}+w_{2}\right) k_{1}^{2}+S q^{1} k_{2}^{2}=0 .
\end{aligned}
$$




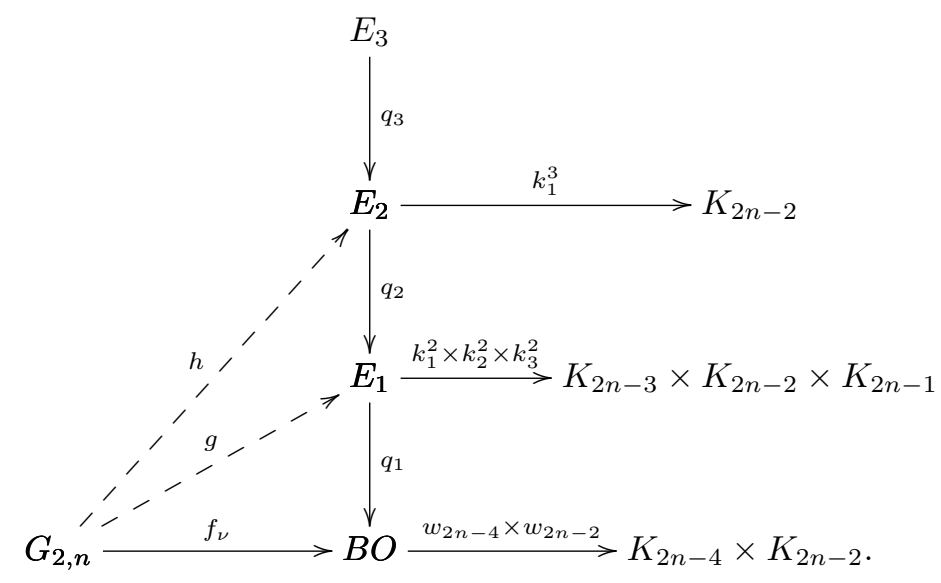

Figure 1: $2 n$-MPT for $p: B O(2 n-5) \rightarrow B O$

Since $\operatorname{dim}\left(G_{2, n}\right)=2 n, f_{\nu}$ lifts up to $B O(2 n-5)$ if and only if it lifts up to $E_{3}$.

By Lemma 3.3(a), $f_{\nu}^{*}\left(w_{2 n-4}\right)=w_{2 n-4}(\nu)=0, f_{\nu}^{*}\left(w_{2 n-2}\right)=w_{2 n-2}(\nu)=0$, so $f_{\nu}$ can be lifted up to $E_{1}$, i.e., there is a map $g_{1}: G_{2, n} \rightarrow E_{1}$ such that $q_{1} \circ g_{1}=f_{\nu}$.

In order to make the next step (to lift $f_{\nu}$ up to $E_{2}$ ), we need to modify (if necessary) the lifting $g_{1}$ to a lifting $g$ such that $g^{*}\left(k_{1}^{2}\right)=g^{*}\left(k_{2}^{2}\right)=g^{*}\left(k_{3}^{2}\right)=0$. By choosing a map $\alpha \times \beta: G_{2, n} \rightarrow K_{2 n-5} \times K_{2 n-3}=\Omega\left(K_{2 n-4} \times K_{2 n-2}\right)$ (i.e., classes $\alpha \in H^{2 n-5}\left(G_{2, n} ; \mathbb{Z}_{2}\right)$ and $\left.\beta \in H^{2 n-3}\left(G_{2, n} ; \mathbb{Z}_{2}\right)\right)$, we get another lifting $g: G_{2, n} \rightarrow E_{1}$ as the composition

$$
G_{2, n} \stackrel{\triangle}{\longrightarrow} G_{2, n} \times G_{2, n} \stackrel{(\alpha \times \beta) \times g_{1}}{\longrightarrow} K_{2 n-5} \times K_{2 n-3} \times E_{1} \stackrel{\mu}{\longrightarrow} E_{1},
$$

where $\triangle$ is the diagonal mapping and $\mu: \Omega\left(K_{2 n-4} \times K_{2 n-2}\right) \times E_{1} \rightarrow E_{1}$ is the action of the fibre in the principal fibration $q_{1}: E_{1} \rightarrow B O$. So, we are looking for classes $\alpha$ and $\beta$ such that $g^{*}\left(k_{1}^{2}\right)=g^{*}\left(k_{2}^{2}\right)=g^{*}\left(k_{3}^{2}\right)=0$. By looking at the relations that produce the $k$-invariants $k_{1}^{2}, k_{2}^{2}$ and $k_{3}^{2}$, we conclude that the following equalities hold (see [3, p. 95]):

$$
\begin{aligned}
& g^{*}\left(k_{1}^{2}\right)=g_{1}^{*}\left(k_{1}^{2}\right)+\left(S q^{2}+w_{2}(\nu)\right)(\alpha) \\
& g^{*}\left(k_{2}^{2}\right)=g_{1}^{*}\left(k_{2}^{2}\right)+\left(S q^{2}+w_{1}(\nu)^{2}+w_{2}(\nu)\right) S q^{1} \alpha+S q^{1} \beta \\
& g^{*}\left(k_{3}^{2}\right)= \begin{cases}g_{1}^{*}\left(k_{3}^{2}\right)+\left(S q^{4}+w_{4}(\nu)\right)(\alpha)+w_{2} \cdot \beta, & n \equiv 3(\bmod 4) \\
g_{1}^{*}\left(k_{3}^{2}\right)+\left(S q^{4}+w_{4}(\nu)\right)(\alpha)+S q^{2} \beta, & n \equiv 1(\bmod 4) .\end{cases}
\end{aligned}
$$

First, we need to prove that the class $g_{1}^{*}\left(k_{1}^{2}\right)$ is in the image of the map $\left(S q^{2}+\right.$ $\left.w_{2}(\nu)\right): H^{2 n-5}\left(G_{2, n} ; \mathbb{Z}_{2}\right) \rightarrow H^{2 n-3}\left(G_{2, n} ; \mathbb{Z}_{2}\right)$. The $k$-invariant $k_{1}^{3}$ is produced by the relation $\left(S q^{2}+w_{2}\right) k_{1}^{2}+S q^{1} k_{2}^{2}=0$ which holds in $H^{*}\left(E_{1} ; \mathbb{Z}_{2}\right)$. Applying $g_{1}^{*}$, we get

$$
\left(S q^{2}+w_{2}(\nu)\right) g_{1}^{*}\left(k_{1}^{2}\right)=S q^{1} g_{1}^{*}\left(k_{2}^{2}\right) .
$$

But, by Lemma 3.6, $S q^{1} g_{1}^{*}\left(k_{2}^{2}\right)=0$. Hence, $g_{1}^{*}\left(k_{1}^{2}\right)$ is in the kernel of the map $\left(S q^{2}+\right.$ 
$\left.w_{2}(\nu)\right): H^{2 n-3}\left(G_{2, n} ; \mathbb{Z}_{2}\right) \rightarrow H^{2 n-1}\left(G_{2, n} ; \mathbb{Z}_{2}\right)$, and according to Lemmas 3.5 and 3.7, this kernel coincides with the image of the map $\left(S q^{2}+w_{2}(\nu)\right): H^{2 n-5}\left(G_{2, n} ; \mathbb{Z}_{2}\right) \rightarrow$ $H^{2 n-3}\left(G_{2, n} ; \mathbb{Z}_{2}\right)$. Thus, we can find a class $\alpha \in H^{2 n-5}\left(G_{2, n} ; \mathbb{Z}_{2}\right)$ such that $g^{*}\left(k_{1}^{2}\right)=0$.

Since $H^{2 n-2}\left(G_{2, n} ; \mathbb{Z}_{2}\right)$ is generated by the classes $w_{1}^{2} w_{2}^{n-2}$ and $w_{2}^{n-1}$ (Corollary 2.8), by Lemma 3.8 and Lemma 3.9 we see that we can choose a class $\beta \in H^{2 n-3}\left(G_{2, n} ; \mathbb{Z}_{2}\right)$ and modify $\alpha$ (by adding, if necessary, the class $\left.w_{1}^{3} w_{2}^{n-4}+w_{1} w_{2}^{n-3}\right)$ to obtain $g$ such that $g^{*}\left(k_{2}^{2}\right)=0$. Since $w_{1}^{3} w_{2}^{n-4}+w_{1} w_{2}^{n-3}$ is in the kernel of the map $\left(S q^{2}+\right.$ $\left.w_{2}(\nu)\right): H^{2 n-5}\left(G_{2, n} ; \mathbb{Z}_{2}\right) \rightarrow H^{2 n-3}\left(G_{2, n} ; \mathbb{Z}_{2}\right)$ (Lemma 3.5), adding this class to the previous $\alpha$ will not spoil the equality $g^{*}\left(k_{1}^{2}\right)=0$.

Finally, observe the class $\beta^{\prime}:=w_{1} w_{2}^{n-2} \in H^{2 n-3}\left(G_{2, n} ; \mathbb{Z}_{2}\right)$. According to Corollary $2.8, w_{2} \cdot \beta^{\prime}=w_{1} w_{2}^{n-1} \neq 0$ and if $n \equiv 1(\bmod 4)$, by Lemma 3.4 ,

$$
S q^{2} \beta^{\prime}=(n-2) w_{1} w_{2}^{n-1}+\left(\begin{array}{c}
n-1 \\
2
\end{array}\right) w_{1}^{3} w_{2}^{n-2}=w_{1} w_{2}^{n-1} \neq 0 .
$$

Since $\beta^{\prime}$ is in the kernel of the map $S q^{1}: H^{2 n-3}\left(G_{2, n} ; \mathbb{Z}_{2}\right) \rightarrow H^{2 n-2}\left(G_{2, n} ; \mathbb{Z}_{2}\right)$ (Lemma 3.8), we can add this class to the previous $\beta$ (if necessary) and obtain a lifting $g$ such that $g^{*}\left(k_{1}^{2}\right)=g^{*}\left(k_{2}^{2}\right)=g^{*}\left(k_{3}^{2}\right)=0$.

Therefore, we can lift $f_{\nu}$ up to $E_{2}$, i.e., there is a map $h_{1}: G_{2, n} \rightarrow E_{2}$ such that $q_{1} \circ q_{2} \circ h_{1}=q_{1} \circ g=f_{\nu}$.

We need to make one more step: to prove that the lifting $h_{1}$ can be modified to a lifting $h$ which lifts up to $E_{3}$, i.e., such that $h^{*}\left(k_{1}^{3}\right)=0$. Arguing as before, we see that it suffices to find classes $a \in H^{2 n-4}\left(G_{2, n} ; \mathbb{Z}_{2}\right)$ and $b \in H^{2 n-3}\left(G_{2, n} ; \mathbb{Z}_{2}\right)$ such that $\left(S q^{2}+w_{2}(\nu)\right)(a)+S q^{1} b=h_{1}^{*}\left(k_{1}^{3}\right) \in H^{2 n-2}\left(G_{2, n} ; \mathbb{Z}_{2}\right)$. But, since $w_{1}^{2} w_{2}^{n-2}$ and $w_{2}^{n-1}$ generate $H^{2 n-2}\left(G_{2, n} ; \mathbb{Z}_{2}\right)$, according to Lemma 3.8 and Lemma 3.10, such classes $a$ and $b$ exist (that is, the indeterminacy of $k_{1}^{3}$ is all of $H^{2 n-2}\left(G_{2, n} ; \mathbb{Z}_{2}\right)$ ). This completes the proof of the theorem.

\section{References}

[1] T. Becker and V. Weispfenning, Gröbner bases: A computational approach to commutative algebra, Graduate Texts in Mathematics 141, Springer-Verlag, New York, 1993.

[2] R. Cohen, The immersion conjecture for differentiable manifolds, Ann. of Math. 122 (1985), no. 2, 237-328.

[3] S. Gitler and M. Mahowald, The geometric dimension of real stable vector bundles, Bol. Soc. Mat. Mex. (2) 11 (1966), 85-107.

[4] M.W. Hirsch, Immersions of manifolds, Trans. Amer. Math. Soc. 93 (1959), no. 2, 242-276.

[5] W.S. Massey, Normal vector fields on manifolds, Proc. Amer. Math. Soc. 12 (1961), no. 1, 33-40.

[6] K.G. Monks, Groebner bases and the cohomology of Grassmann manifolds with application to immersion, Bol. Soc. Mat. Mex. (3) 7 (2001), no. 1, 123-136.

[7] F. Nussbaum, Obstruction theory of possibly nonorientable fibrations, Northwestern Unversity Ph.D. thesis (1970). 
[8] V. Oproiu, Some non-embedding theorems for Grassmann manifolds $G_{2, n}$ and $G_{3, n}$, Proc. Edinburgh Math. Soc. 20 (1976-77), no. 3, 177-185.

[9] T.A. Shimkus, New immersions of Grassmann manifolds, Bol. Soc. Mat. Mex. (3) $\mathbf{1 3}$ (2007), no. 2, 381-389.

Zoran Z. Petrović zoranp@matf.bg.ac.rs

University of Belgrade, Faculty of Mathematics, Studentski trg 16, Belgrade, Serbia

Branislav I. Prvulović bane@matf.bg.ac.rs

University of Belgrade, Faculty of Mathematics, Studentski trg 16, Belgrade, Serbia 